
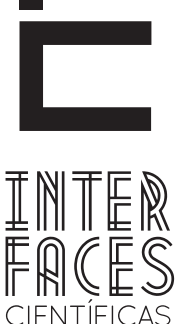

HUMANASE SOCIAIS

\title{
PLANEJAMENTO ESTRATÉGICO SITUACIONAL APLICADO EM UMA PEQUENA EMPRESA DE SERVIÇOS DE ITAPEVA, SP
}

\author{
Jorge Luiz Knupp Rodrigues ${ }^{1}$ \\ Neusa Maria Bastos Fernandes Santos ${ }^{3}$
}

Oderlei Ferreira dos Santos²

\section{RESUMO}

Este trabalho visa identificar quais os resultados de um diagnóstico sobre a gestão estratégica de uma pequena empresa do setor de serviços da cidade de Itapeva, SP. Foi realizado um estudo de caso, por meio da aplicação da Análise Estratégica Tridimensional descrita por Santos (2014), que visa identificar como se desenvolvem as ações estratégicas na organização, tendo como embasamento os modelos de Planejamento Estratégico Tradicional e Planejamento Estratégico Situacional. A relevância do estudo consiste no tocante que as micro e pequenas empresas possuem grandes dificuldades em institucionalizar métodos de planejamento estratégico levando a problemas de competitividade. Os resultados demonstram que embora não tenha um método formal de planejamento estratégico instituído, a empresa desenvolve diversas ações estratégicas que a mantém atuante no mercado e que a gestão pode ser ajustada em alguns aspectos para aprimorar os conceitos estratégicos praticados.

\section{PALAVRAS-CHAVE}

Gestão estratégica. Planejamento estratégico situacional. Pequenas empresas. Diagnóstico estratégico. 


\section{ABSTRACT}

This paper aims to identify the results of an assessment of the strategic management of a small business service sector of the city of Itapeva, SP. We conducted a case study, through the application of the Strategic Tridimensional described by Santos (2014), which aims to identify and develop strategic actions in the organization, with the basement models Traditional Strategic Planning and Situational Strategic Planning. The relevance of the study is regarding the micro and small companies have great difficulties in institutionalizing strategic planning methods leading to competitiveness problems. The results show that although no formal method of strategic planning established, the company develops various strategic actions that keeps it active in the market and that management can be adjusted in some ways to enhance the strategic concepts practiced.

\section{KEYWORDS}

Strategic Management. Situational Strategic Planning.Small Businesses.Strategic Diagnosis.

\section{RESUMEN}

Este trabajo tiene como objetivo identificarlos resultados de una evaluación de la gestión estratégica de una pequeña empresa del sector servicios en la ciudad Itapeva SP. Un estudio de caso, mediante la aplicación del Análisis Estratégico Dimensional descrito por Santos(2014), cuyo objetivo es identificar y desarrollar acciones estratégicas en la organización, teniendo como base los modelos de Planificación Estratégica Tradicionaly Planificación Estratégica Situación a la que se llevó a cabo. La relevancia del estudio es en relación al micro y pequeñas empresas tienen grandes dificultades en los métodos de planificación estratégica que conducen a los problemas de competitividad institucionalización. Los resultados muestran que aunque no es un método formal de planificación estratégica establecida, la compañía ha desarrollado varias acciones estratégicas que se mantiene activo en el mercado y que la gestión se puede ajustaren algunas formas de mejorar los conceptos estratégicos practicados.

\section{PALABRAS CLAVE}

Gestión Estratégica. Planificación Estratégica Situacional. Las pequeñas empresas. Diagnóstico estratégico. 


\section{INTRODUÇ̃̃O}

Autores como Longenecker, Moore e Petty (1997), Terence (2002) e Chér (1991), apontam características das pequenas empresas, situando-as como importantes atores econômicos, social e produtivo. No Brasil, isso pode ser corroborado por dados das Micro e Pequenas Empresas (MPE) descritos pelo Serviço Brasileiro de Apoio às Micro e Pequenas Empresas - SEBRAE (2011) que apresenta um panorama geral das MPE e o Instituto Brasileiro de Geografia e Estatística - IBGE (2011) que demonstram uma representatividade acima de $99 \%$ no cenário de empreendimentos nacionais. De acordo com o IBGE (2003), no ano de 2001, constatou-se que $97,6 \%$ dessas empresas são do setor de serviços e comercio.

As pequenas empresas possuem um sério problema que consiste em sua sustentabilidade, pois conforme o SEBRAE, nos anos de 2008 e 2010, respectivamente, $62 \%$ e $58 \%$ das MPE findaram antes de completar seis anos (SEBRAE, 2008 e 2010) e uma das causas apontadas, corroborando com estudos apresentados por Trejo (2010) e Quadros e outros autores (2012), está relacionada a problemas de falha ou falta de uma gestão estratégica eficiente.

Cabe destacar que a gestão estratégica se faz essencial na gestão das empresas. De acordo com Oliveira (2001), o planejamento contribui com os objetivos da empresa e a estratégia relaciona-se com a melhor forma de utilizar os recursos para obter os resultados, entretanto, Quadros e outros autores (2012) apontam que as MPE não conseguem alavancar resultados com a implantação do planejamento estratégico. Mintzberg (2004) aponta que o modelo clássico da ferramenta acumula fragilidades na complexidade das análises, inadequação do plano e falhas administrativas na implementação.

O economista chileno Carlos Matus, na tentativa de adequação do Planejamento Estratégico em instituições públicas, desenvolveu o Planejamento Estratégico Situacional (PES), como uma alternativa ao Planejamento Estratégico Tradicional. 0 novo método, basicamente composto por quatro momentos distintos, considera a atuação dos diversos atores no jogo social e estabelece um método flexível às diversas alterações da realidade em busca dos objetivos e pressupõe constante adaptação a cada situação concreta em que é aplicado.

Dado os aspectos das MPE, do Planejamento Estratégico e tendo o PES como uma nova abordagem entre os métodos de gestão estratégica, este trabalho busca analisar a gestão estratégica à luz do PES em uma pequena empresa de serviços da cidade de Itapeva, SP. Para a realização do trabalho levou-se em consideração a Análise Estratégica Tridimensional descrita por Santos (2014), a qual considera o grau de participação, utilidade e importância dos momentos do PES na gestão das MPE, tendo em vista a identificação das práticas estratégicas adotadas na empresa e pontos que podem ser melhorados na gestão que facilite ao empreendedor a tomada de decisão e a gestão no dia a dia em alternativa ao Planejamento Estratégico Tradicional.

\section{METODOLOGIA}

Para Marconi e Lakatos (2010) o delineamento da pesquisa é definido como estudo de caso comparativo com objetivo exploratório que visa uma análise empírica e teórica, pois trata de relações em que o fator humano se faz presente e busca entender a situação e sua complexidade em uma realidade diante da exaustão de um objeto delimitado.

A abordagem da pesquisa é considerada como qualitativa e quantitativa, pois levanta as opiniões e atitudes de pessoas envolvidas na gestão da empresa estudada e analisa as interações dos aspectos identificados, tendo por base o referencial teórico a fim de compreender o processo estratégico, que neste caso se deu pelo estudo de uma empresa do setor de serviços da cidade de Itapeva, SP, sendo abordado na 
ocasião, o proprietário e um funcionário que ocupa a função de gestor e auxilia o proprietário na gestão estratégica. Para a realização do estudo foi considerada a Análise estratégica tridimensional descrita por Santos (2014) que possui a finalidade de mensurar as contribuições do PES para a gestão das MPE.

Conforme a Figura 1, a Análise Estratégica Tridimensional representa o grau de contribuição do PES em comparação com o Planejamento Estratégico Tradicional, correlacionando tal variável com a utilidade e importância na gestão.

Figura 1 - Análise estratégica tridimensional

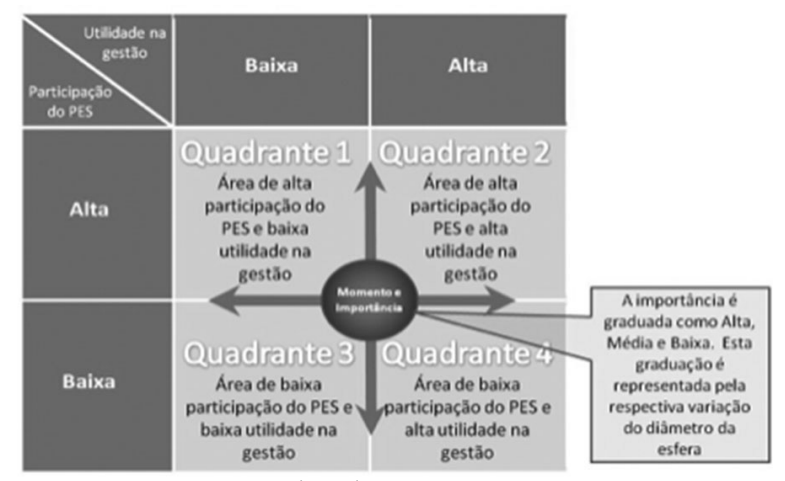

Fonte: Adaptado de Santos (2014),

Na Figura 1, pode-se observar o posicionamento dos momentos do PES, representado pela esfera, demonstra utilidade e participação na gestão. A utilidade busca demonstrar o quanto um determinado momento do PES é útil para a gestão da MPE, ou seja, que tem serventia e vantagens no uso. No plano horizontal quanto mais a direita estiver a esfera do momento, maior é a utilidade. Este posicionamento é resultante do grau de utilidade, de zero a dez, que foi atribuído pelo gestor do empreendimento para cada momento do PES.

A participação do PES na gestão (eixo vertical) tem por finalidade expressar o quanto o PES, por meio de seus distintos momentos, está presente na gestão da empresa pesquisada em comparação com os aspectos do Planejamento Estratégico Tradicional. Para tanto, foi aplicado um questionário com base nos conceitos de Ansoff e outros autores (1981), Porter (2004), Mintzberg (2004), Mintzberg e outros autores (2006), Peng (2008), e Oliveira (2001), e em referências sobre o PES conforme Huertas (1996) e Matus (1996, 1996b, 1997 e 2006).

Os posicionamentos da utilização foram obtidos por meio da proporção de utilização dos conceitos do PES, considerando as respostas dos questionários aplicados junto ao gestor e o funcionário que o auxilia na gestão estratégica, considerando o cálculo expresso como demonstrado pela Figura 2.

Figura 2 - Expressão para o cálculo da participação do PES

$$
\text { Participação do PES }(\%)=\frac{R P E S}{R P E} \times 100
$$

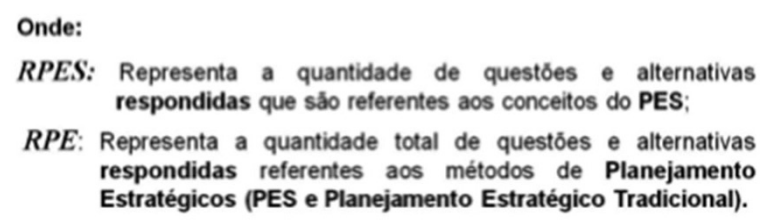

Fonte: Santos (2014).

A importância de cada momento para a gestão é tratada na terceira dimensão levantada. Em outras palavras, busca graduar o grau de relevância dos momentos para a MPE. Esta importância é representada pelo respectivo diâmetro da esfera que representa o momento, isto é, quanto maior a esfera, maior é a importância que ela representa. A dimensão do diâmetro da esfera revela de forma não proporcional a graduação da importância, podendo ser importância alta, média ou baixa.

A graduação da importância foi obtida pelo valor, de zero a dez, atribuído para cada momento do PES pelo gestor do empreendimento. De acordo com Santos (2014) a intenção da análise estratégica tridimensional consiste em demonstrar a forma de contribuição do 
PES na gestão estratégica das MPE e em apresentar um diagnóstico da prática da gestão estratégica podendo assim determinar ajustes institucionais e apoiar a adequação da utilização dos métodos de gestão.

\section{RESULTADOS E DISCUSSÕES}

As pequenas e micro empresas respondem com $20 \%$ de todo o Produto Interno Bruto do Brasil. Consta ainda que essas empresas situam-se em diversos nichos de negócios, sendo 58\% no comércio, 32\% empresas de serviços, $9 \%$ na indústria e 0,4\% na agropecuária de acordo com o SEBRAE (2008). O SEBRAE e DIEESE (2011) apontam a evolução do número de empregos gerados pelas MPE, saindo de 8,6 milhões de empregos em 2000 para 14,7 milhões de empregos em 2010, sendo que nesse ano foi responsável por $99 \%$ dos estabelecimentos de empregos, $51,6 \%$ dos empregos e $39,7 \%$ da massa de remuneração. O SEBRAE (2013b) destaca o crescimento do número de pequenas empresas ao mencionar o acréscimo de $26 \%$ de optantes do regime fiscal Simples Nacional em dezembro do ano de 2012 em comparação com o mesmo período de 2011.

Santos, Carvajal Júnior e Crivelaro (2012), referindo-se ao setor de serviços, afirmam que há um crescente aumento da demanda e oferta de serviços, seja pelos avanços tecnológicos ou alteração do comportamento da população, como o aumento da inserção da mulher no mercado de trabalho ou o aumento da urbanização.

Longenecker, Moore e Petty (1997) enfatizando a gestão das MPE, apontam que a estratégia consiste em um processo complexo que necessita do compromisso do empreendedor. Rodrigues, Santos e Batista (2011) apontam que o Planejamento Estratégico é pouco utilizado nas MPE devido à elevada necessidade de especialização para a análise de informações e tomadas de decisões. Oliveira, Terence e Escrivão Filho (2008) apontam que o Planejamento Estratégico não se faz suficiente para alavancar os resultados das MPE.
O Planejamento Estratégico consiste em uma das ferramentas de gestão mais utilizada entre os executivos, mesmo quando se trata de resultados em curto prazo, afirmam Rigby e Bilodeau (2009). O planejamento estratégico não se separa da gestão é o que afirmam Latif e outros autores (2013). Para Fischmann e Almeida (2009) a administração empresarial possui o nível operacional, que cuida de operações como produção, compras e vendas; o nível administrativo que trata da integração e relações da empresa, como finanças e recursos humanos, e o nível estratégico, que orienta a organização em seu ambiente com o objetivo de obter uma organização eficaz, o que significa, conforme Oliveira (2001), produzir alternativas criativas, maximizar recursos, obter resultados e aumentar o lucro.

A partir dos anos de 1960, como alternativa ao método tradicional de Planejamento Estratégico, inicia-se a concepção do método PES para ser aplicado nas instituições públicas de forma a suprir as especificidades deste tipo de instituição, as quais não eram atendidas com o método tradicional. Considerando a experiência na área política-econômica e planejamento, em consequência de discussões sobre o formalismo, neutralidade e efetividade do planejamento, enquanto membro da Comissão Econômica para a América Latina e o Caribe (CEPAL), Matus (1972 apud BELCHIOR, 1999) constrói o PES como um método próprio com base em sua formação e experiência na área de planejamento.

O PES rejeita o determinismo, como prazos rígidos e agendas. 0 método opta por cálculos estratégicos, desconfia das previsões e atua em um jogo interativo em constante mudança. O PES considera que planejamento é estabelecido pela ação política e que o planejamento não situa no futuro, pois isso está em constante construção. O autor considera que o PES atua por problema e de forma geral, pode ser instituído em problemas que derivam projetos, e que por sua vez, requer uma gestão operacional (GALETAR, 2013).

É possível a observação de distinções entre o Planejamento Estratégico Tradicional e o PES, e uma das carac- 
terísticas atribuída estabelece que o PES se concretiza na ação, o que implica em sua concepção, execução, controle e revisão conforme as consequências. Outra distinção que pode ser atribuída remete ao modelo de planejamento e sua aplicação. Matus (1997) afirma que o PES é um método para um sistema de incerteza dura (sistema complexo) e aponta o planejamento estratégico tradicional como instrumento determinista puro e estocástico.

O PES consiste na composição de quatro momentos: a apreciação situacional por meio da explicação da realidade, apostas em cenários na concepção do plano, análise estratégica para viabilizar o plano e cálculo, ação e correção para atuar no cotidiano (HUERTAS, 1996). Os quatro momentos do PES podem ser resumidos em questões básicas que também o diferencia do planejamento estratégico tradicional, conforme apresentado no Quadro 1.

Quadro 1 - Comparativo entre o PES e o Planejamento Estratégico Tradicional

\begin{tabular}{ccc}
\multicolumn{1}{c}{ Questão } & $\begin{array}{c}\text { Planejamento } \\
\text { Estratégico } \\
\text { Tradicional }\end{array}$ & $\begin{array}{c}\text { Planejamento } \\
\text { Estratégico } \\
\text { Situacional }\end{array}$ \\
$\begin{array}{l}\text { Como explicar } \\
\text { a realidade? }\end{array}$ & Diagnóstico & $\begin{array}{l}\text { Apreciação si- } \\
\text { tuacional }\end{array}$
\end{tabular}

Como conce- Cálculo paramé- Apostas
ber o plano? trico

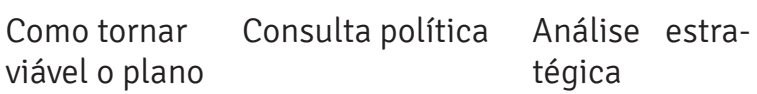

necessário?

$\begin{array}{lll}\text { Como agir a } & \text { Execução do pla- } & \text { Cálculo ação e } \\ \text { cada dia de } & \text { no } & \text { correção }\end{array}$ forma planejada?

Fonte: Huertas (1996, p. 99), adaptado pelos autores.
O planejamento estratégico situacional concentra as ações para uma realidade em constante alteração. Neste sentido, o plano constitui-se em uma aposta. Pode-se planejar e não prever o futuro, porque a realidade está cheia de surpresas que impedem a predição. Pode-se prever com algumas variáveis, mas os planos de contingência são indispensáveis para enfrentar as surpresas, conforme Migliato (2004). Segundo Rivera (2011), trata-se de uma aposta argumentativa. Conforme o autor, diante de problemas não estruturados o plano constrói sua aceitabilidade social e confiabilidade nos conceitos de processos argumentativos que se aproximam de um modelo de discussão crítica de natureza dialética.

Matus (2006) afirma que considerando o jogo social, o ator escolhe o plano, mas não pode escolher as circunstâncias em que este será aplicado, dessa forma, o autor propõe a aplicação da técnica de cenários e técnicas de absorção de incertezas sobre as variáveis e o estabelecimento de planos de contingência.

Considerando as questões de Quadros e outros autores (2004) e Oliveira, Terence e Escrivão Filho (2008) sobre as dificuldades das MPE com o método do planejamento estratégico tradicional, aliado às características e fragilidades apontadas por Mintzberg (2004) e a emergência do PES, de acordo com o método descrito, Santos (2014) propõe a Análise Estratégica Tridimensional como um diagnóstico estruturado para obter informações da gestão da organização estudada, bem como um método para apoiar os gestores à definirem ajustes na gestão estratégica de acordo com as particularidades da organização, levando em consideração os diversos preceitos teóricos.

Tendo este pressuposto teórico, o presente estudo ocorreu em uma empresa fundada no ano de 2001 e situada na região central de Itapeva, SP. De acordo com o SEBRAE e DIEESE (2011), trata-se de uma pequena empresa, pois possui 27 funcionários e atua no setor de prestação de serviços. Esta classificação é reforçada por Santos e Veiga (2012) ao considerar o faturamento no ano de 2013 entre R\$ 360 mil e R\$ 3.6 milhões. 
A referida empresa destina-se a fornecer serviços de consultoria em engenharia, medicina ocupacional e clínica médica, a qual engloba programa de controle médico de saúde ocupacional, programa de conservação auditiva, programa de prevenção respiratória, laudo ergonômico; segurança e saúde no trabalho na agricultura; segurança e saúde no trabalho em estabelecimento de saúde; perfil profissiográfico previdenciário, formação de comissão interna de prevenção de acidentes, programa de prevenção de riscos ambientais; laudo de insalubridade, laudo de periculosidade; programa de condições e meio ambiente de trabalho na indústria da construção; mapa de riscos, treinamentos voltados à saúde e segurança ocupacional, planos de saúde e clínica médica multiespecializada.

Possui uma carteira com mais de 900 contratos assinados (pessoas físicas e jurídicas), o que representa mais de 15 mil pessoas que se situam na cidade de Itapeva e região. Trata-se da empresa líder na região, pois agrega em torno de $75 \%$ dos clientes em potencial. 0 proprietário da empresa atua tanto nas funções operacionais quanto nas funções administrativas, sendo ele 0 principal precursor das estratégias. 0 processo decisório, também, conta com o apoio de um profissional que trabalha na gerência da empresa e auxilia o proprietário no processo decisório. Nos seus quase 13 anos de existência, a empresa passou por períodos críticos como a falta de clientes, desconhecimento e incertezas de mercado, bem como crises econômicas entre outros fatores.

A empresa, para o êxito nos negócios, adotou diversas estratégias e táticas, como a observação do ambiente e inserção de ações em momentos oportunos, a preparação e profissionalização da função administrativa, diversificação e lançamentos de novos serviços, identificação e satisfação das necessidades dos clientes e implantação de ferramentas como procedimentos operacionais e pipeline de vendas e formalização das ações a se realizar em curto prazo. Visando a sustentabilidade do negócio e resultados positivos futuros, atualmente a empresa passa por um estagio estratégico de reorganização administrativa em busca de uma melhor estrutura para diversificar e lançar novos produtos, dentre eles, a reformulação dos planos de saúde vendidos e a inovação para o lançamento de novos serviços.

Com a aplicação da Análise Estratégica Tridimensional, embasado nos preceitos de Porter (2004), Mintizberg (2004), Mintizberg e outros autores (2006), Peng (2008), Oliveira (2001) Huertas (1996) e Matus (1996, 1996b, 1997 e 2006), levando em consideração a estrutura e métodos de gestão, aspectos de planejamento e as principais ferramentas utilizadas na empresa, foi possível constatar as seguintes características na empresa:

- Possui uma estrutura gerencial com recursos e métodos para suportar a administração da empresa, como por exemplo, gestão financeira e gestão de recursos humanos. 0 empreendedor relatou que a implantação da estrutura gerencial foi possível com o crescimento da empresa. Tal estrutura resultou na implantação de métodos sistêmicos de gestão que fornecem o que empreendimento necessita para sua sobrevivência e competitividade;

- Não possui uma sistemática de planejamento estratégico estruturado, da mesma forma que não formaliza um plano estratégico, porém, ações estratégicas, ocorrem informalmente;

- Possui um planejamento de curto prazo de ações operacionais e uma visão de longo prazo.

Conforme o gestor da empresa, a missão e os valores estabelecidos servem como um guia para as estratégias, porém o estabelecimento da missão e da visão só foi possível com a evolução e amadurecimento da empresa. A missão, a visão e os valores foram oriundos do estágio de maturação da empresa e nem todas as estratégias provém da missão e dos valores.

Sobre a definição de objetivos e metas, a empresa possui sistemática estabelecida para o seu desdobra- 
mento, porém, esses objetivos e metas não possuem relação direta com programas estratégicos provenientes da missão. Trata-se de metas operacionais de curto prazo, essenciais, à sobrevivência do negócio, como por exemplo, a meta número de visitas a clientes. Os planos estratégicos consistem em aspirações e visões de longo prazo que oportunamente trasformam-se em planos de ações exequíveis em curto prazo.

Na pesquisa realizada na empresa foi observado que a análise situacional da mesma ocorre de forma dinâmica e engloba perspectivas internas e externas. Este processo é dirigido pelo empreendedor de maneira informal, porém, conforme Panagiotou e van Wijnen (2005), consideram conceitos teóricos adaptados a prática e serve como um pano de fundo para a realização de análises e formular ações.

Observa-se, também, que a empresa realiza analise ambiental (interna e externa), de forma dinâmica, não estruturada e dessa forma, deriva-se planos para a resolução de problemas e o alcance de oportunidades, como por exemplo, a venda de um mix de serviços diferenciado com base na característica do cliente. Assim, o planejamento se dá de maneira formal somente para as ações operacionais de curto prazo, o que, conforme Peng (2008) contribui para o atendimento dos objetivos. A empresa busca um planejamento flexível e dessa forma sobressaem ações emergentes (MINTBERG, 2004).

Cabe destacar também que a prioridade do planejamento consiste no curto prazo, isso devido às grandes variações e alterações ambientais em curto espaço de tempo, obrigando a empresa ser ágil e precisa na tomada de decisão para se adaptar as constantes alterações do ambiente em que se situa. A concepção do plano não leva em considerações predições, mas sim a análise de cenários, tendo dessa forma a atuação em várias frentes e a aposta em uma situação que pode requerer a viabilidade do plano. O conceito converge com Matus (1996a) que considera cálculos incertos que exigem a atuação do planejamento em varias frentes, tendo a possibilidade de revisão ou a formulação de novos planos.
No que diz respeito aos aspectos financeiros e de marketing, estes são demandados pelas estratégias emergentes oriundos dos problemas e oportunidades do ambiente. Há uma sistematização dos aspectos operacionais, como o fluxo de caixa e pipeline de vendas, porém tais ferramentas, entre outras, foram implementadas de forma gradativa de acordo com a evolução da maturidade da empresa. Para a implantação das estratégias (mesmo que ela seja informal), o empreendimento busca interagir com os atores que possam interferir no resultado, como por exemplo, o estabelecimento de parcerias e compartilhamento de resultados com os funcionários. Considerando as características da empresa, e a aplicação do método proposto, foi possível planificar Análise Estratégica Tridimensional conforme a Figura 3.

Figura 3 - Planificação da análise estratégica tridimensional

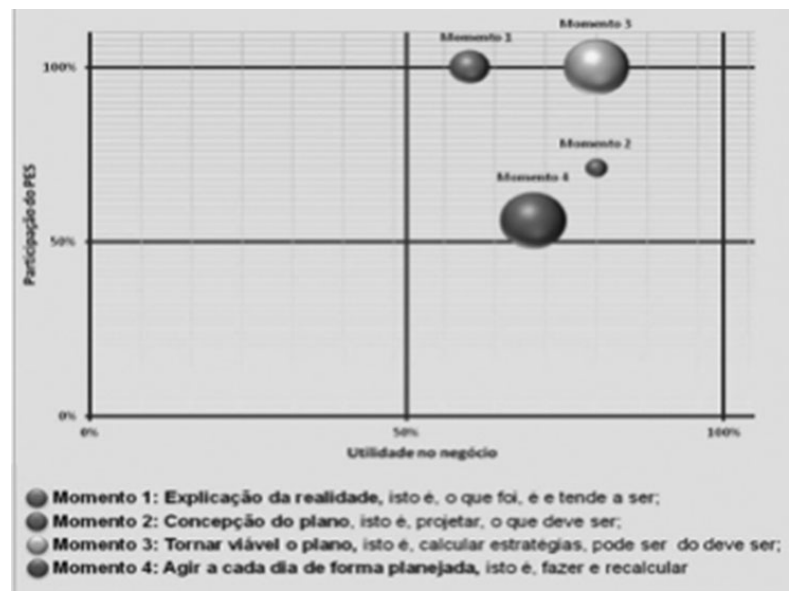

Fonte: Dados da pesquisa, com base em Santos (2014).

Observa-se que o detalhamento da análise demonstra que o empreendimento possui uma característica versátil, pois busca a implantação de novos serviços bem como a renovação de seus processos. Esses fatores motivaram a participação de $100 \%$ dos conceitos do PES nos Momentos 1 e 3, pois além da busca pelo entendimento da realidade para deman- 
dar ações, há também o anseio por implementar as ações, que podem apresentar inviáveis em um primeiro momento, porém, com ações de articulação dos atores do cenário, pode ser viabilizada em um segundo momento.

0 resultado de $71 \%$ no Momento 2 é devido a empresa se embasar em dados estatísticos para a tomada de decisão, porém esses dados possuem mais intensidade nas decisões do nível operacional, como a medição de desempenho do que em momentos estratégicos, como a decisão de lançar um novo serviço. 0 Momento 4, com menor graduação (56\%), se deu pela prevalência da decisão do proprietário frente as novas prioridades do dia a dia, prejudicando assim, a execução do previsto.

Sobre os resultados referentes à utilidade, também são justificados pelo perfil do empreendimento. Os Momentos 2 e 3, ambos com $80 \%$ e o Momento 4 com $70 \%$ é devido a busca para identificar e implantar ações para a expansão da empresa e aumentar a competitividade. A menor graduação no Momento 1 com $60 \%$, está atrelada à busca da empresa em aprimorar seus controles de gestão para embasar a tomada de decisão em dados estatísticos.

Já as graduações de importância tiveram influência da maturidade da empresa. Passados 12 anos no mercado, a empresa acumula aprendizados empíricos que levam a valoração dos aspectos de gestão. As maiores graduações foram obtidas nos Momentos 3 e 4 pela busca em implementar as ações, e os Momentos 1 e 2 , com respectivos $80 \%$ e $70 \%$ provém da falta de aderência dos planos previstos, seja nas ações ou prazos, com a prática efetivada.

Em síntese, pode-se constatar por meio do estudo, que a empresa abordada possui uma gestão dinâmica para viabilizar ações em busca de seus objetivos, e para tanto, procura-se embasar em dados estatísticos para a tomada de decisão no nível operacional. No nível estratégico prevalece a análise do gestor mediante a prioridade que se altera constantemente, com isso, há uma baixa aderência aos planos previstos. Assim, destacam-se pontos positivos na gestão, como a flexibilidade da empresa para aproveitar oportunidades não previstas e a viabilização de objetivos potenciais, por outro lado, denota pontos a melhorar, como a transformação de dados em informações para apoiar as decisões estratégicas.

\section{CONCLUSÃO}

Uma das contribuições deste estudo relaciona-se ao fato da possibilidade de conhecer a empresa estudada, a qual passou por um processo de evolução com o transcorrer do tempo. 0 empreendedor aprimorou as habilidades e estrutura de gestão organizacional no sentido atender as aspirações do proprietário, fazendo disso um diferencial competitivo para o negócio em momentos de crise e para o aproveitamento de oportunidades.

Já com a utilização da Análise Estratégica Tridimensional foi possível diagnosticar a gestão estratégica na empresa, que em síntese, se dá pelo planejamento de cenários, entendimento sobre o que significa a minha realidade para os stakeholders e a viabilização do plano para sua implementação por meio de trabalho com os atores de influência, e assim, com prioridades e ações cambiantes, busca-se a manutenção e o aumento da competitividade por meio de um planejamento com horizonte de curto prazo.

Levando-se em consideração os modelos de Planejamento Estratégico abordados, não foi constatado um modelo implantado na íntegra, entretanto, constatou-se a contribuição de vários aspectos do PES e Planejamento Estratégico Tradicional que auxiliam a empresa. Dessa forma, é essencial que tais aspectos, como por exemplo, a análise ambiental e elaboração de planos de ação, sejam fortalecidos, pois podem significar manutenção da vantagem competitiva. 
Conforme apresentado na Figura 3, o Diagnóstico resultante da planificação tridimensional possibilitou destacar aspectos que podem ser ajustados na gestão, como a possibilidade de melhorar a gestão com dados estatísticos como insumo de análise de dados para a tomada de decisão, a consideração e formalização das ações emergentes como ações estratégicas válidas, quando em seu estabelecimento forem consideradas análises estratégicas, tendo dessa forma, uma maior credibilidade na gestão estratégica e um melhor aprendizado de como agir no dia a dia em uma realidade cambiante.

Implantação e/ou sistematização de um modelo de planejamento estratégico na íntegra, conforme os preceitos teóricos podem não representar resultados práticos como o equilíbrio estratégico, ou seja, a aplicação racional dos conceitos teóricos à prática, pois a empresa possui diversas especificidades, dentre elas, a própria estrutura que é adversa à adoção de determinados aspectos preconizados nas teorias, entretanto, o conhecimento e o pensamento estratégico aliado aos fatores empíricos podem trazer ganhos ao empreendedor.

\section{REFERÊNCIAS}

ANSOFF, H.I. Estratégia empresarial. São Paulo: McGraw do Brasil, 1977.

BELCHIOR, M. A aplicação de planejamento estratégico situacional em governos locais: possibilidades e limites - os casos de Santo André e São José dos Campos. 1999. Dissertação (Mestrado em Administração) - Fundação Getúlio Vargas, Escola de Administração de Empresas de São Paulo, São Paulo, 1999.

BONACIM, C.A.G; CUNHA, J.A.C.; CORRÊA, H.C. Mortalidade dos empreendimentos de micro e pequenas empresas: causas e aprendizagem. Gestão\&Regionalidade, São Caetano do Sul, v.25, n.74, 2009, p.60-78.
BORDUM, A. The strategic balance in a change management perspective. Society and Business Review, v.5, n.3, 2010, p.245-258.

CHÉR. R. A gerencia das pequenas e médias empresas: o que saber para administrá-las. 2.ed. São Paulo: Maltense, 1991.

FIGUEIREDO FILHO, W.B.; MÜLLER, G. Planejamento estratégico segundo Matus: proposta e crítica. In.: GERARDI, L.H.O; MENDES, I.A. (Orgs) Do natural, do social e suas interações. [online]. Rio Claro, 2002, p. 121-132. Disponível em <http://www.rc.unesp.br/igce/geografia/ pos/dowdonatural.php>. Acesso em: 5 out. 2012.

FISCHMANN, A.A.; ALMEIDA, M.I.R. Planejamento estratégico na prática. São Paulo: Atlas, 2009.

HUERTAS, F. 0 método PES: entrevista com Matus. São Paulo: Fundap, 1996.

IBGE - Instituto Brasileiro de Geografia e Estatística, Coordenação de Serviços e Comércio. As micro e pequenas empresas comerciais e de serviços no Brasil 2001. Rio de Janeiro: IBGE, 2003.

LONGENECKER, J.G.; MOORE, C.W.; PETTY, T.W. Administração de pequenas empresas: ênfase na gerência empresarial. São Paulo: Makron Books, 1997.

MACHADO, J.R. Planejando a estratégia de pequenos negócios. Rio de Janeiro: Qualitymark, 2005.

MARCONI, M. A.; LAKATOS, E. M. Fundamentos de metodologia científica. 7.ed. São Paulo: Atlas, 2010.

MATUS, C. O plano como aposta. In: GIACOMONI, J.; PAGNUSSAT, J. L. Planejamento e orçamento governamental: coletânea. Brasília: Enap, 2006. v.2.

MATUS, C. 0 Método PES: Roteiro de análise teórica. São Paulo: Fundap, 1997. 
MATUS, C. Adeus, senhor presidente. São Paulo: Fundap, 1996a.

MATUS, C. Estratégias políticas: chimpanzé, Maquiavel e Gandhi. São Paulo: Fundap, 1996 b.

MIGLIATO, A. L. T. Planejamento estratégico situacional aplicado à pequena empresa: estudo comparativo de casos em empresas do setor de serviços (hoteleiro) da região de Brotas-SP. 2004. Dissertação (Mestrado em Engenharia de Produção) - Escola de Engenharia de São Carlos, Universidade de São Paulo, São Carlos, 2004. Disponível em: <http://www.teses.usp.br/teses/disponiveis/18/18140/tde-24112004-073829/>. Acesso em: 16 set. 2012.

MINTZBERG, H. et al. $\mathbf{0}$ processo da estratégia: conceitos, contextos e casos selecionados. 4.ed. Porto Alegre: Bookman, 2006.

MINTZBERG, H. Ascenção e queda do planejamento estratégico. 4.ed. Porto Alegre: Bookman, 2004.

OLIVEIRA, D. P. R. Planejamento Estratégico: conceitos, metodologia e práticas. 16.ed. São Paulo: Atlas, 2001.

OLIVEIRA, J.;TERENCE, A.C.F.; ESCRIVÃO FILHO, E. Planejamento estratégico e operacional na pequena empresa: impactos da formalização no desempenho e diferenças setoriais. In: Encontro Anual da Anpad, 32. Anais... Rio de Janeiro: Anpad, 2008.

PANAGIOTOU, G., \& van WIJNEN, R. The "telescopic observations" framework: An attainable strategic tool. Marketing Intelligence \& Planning, v.23, n. 2, 2005, p.155-171.

PENG, M.W. Estratégia global. São Paulo: Thomson Learning, 2008.

PORTER, M. E. Estratégia Competitiva: técnicas para análise da indústria e da concorrência. 2.ed. Rio de Janeiro: Elsevier, 2004.
QUADROS, J. N. et al. Planejamento estratégico para pequena empresa: um estudo de caso em uma pequena empresa de Santa Maria/RS. Revista da Micro e Pequena Empresa, Campo Limpo Paulista, v.6, n.2, 2012, p.71-88.

RIGBY, D.; BILODEAU, B. Management Tools and Trends. Bain \& Co., 2009, Disponível em: <www.joinbainaustralia.com/bainweb/PDFs/cms/Public/Management_Tools_2009.pdf>. Acesso em: 5 mar. 2013.

RODRIGES, J.L.K.; RODRIGUES, C.E.M. Marketing de serviços. In: SANTOS, F.A.; JÚNIOR, C.J.C.; CRIVELARO, M. (Orgs). Administração de empresas no setor de serviços. São Paulo: Porto de Ideias, 2012.

RODRIGUES, J.L.K.; SANTOS, I.C.; BATISTA, F.C. 0 processo estratégico em empresas de pequeno porte: uma ferramenta de apoio à gestão e ao crescimento de empreendimentos. Rio de Janeiro: Publit, 2011.

SANTOS, Oderlei Ferreira dos. Contribuições do planejamento estratégico situacional para a gestão de micro e pequena empresas: um estudo de caso comparativo em Itapeva, SP. Dissertação (Mestrado) - Universidade de Taubaté, Taubaté, 2014.

SANTOS, F.A.; JÚNIOR, C.J.C.; CRIVELARO, M. (Orgs). Administração de empresas no setor de serviços. São Paulo: Porto de Ideias, 2012.

SANTOS, F.A.; VEIGA, W, E. Contabilidade: com ênfase em micro, pequenas e médias empresas. 2.ed. São Paulo: Atlas, 2012.

SEBRAE (org.); DIEESE. Anuário do trabalho na micro e pequena empresa 2010-2011. 4.ed. Brasília: DIEESE, 2011.

SEBRAE. 10 anos de monitoramento da sobrevivência e mortalidade das empresas. São Paulo: SEBRAE, 2008. 
TERENCE. A.C.F. Planejamento Estratégico como ferramenta de competitividade na pequena empresa: desenvolvimento e avaliação de um roteiro para o processo de elaboração do planejamento. Dissertação (Mestrado em Engenharia de Produção) - Universidade de São Paulo, Escola de Engenharia de São Carlos, São Carlos, 2002.
TREJO, M. D., 0 planejamento estratégico e as pequenas empresas: desafios e possíveis alternativas. Artigo apresentado ao Curso de Pós-Graduação em Administração da UNIFAMMA - Faculdade Metropolitana de Maringá, MBA em Consultoria Organizacional com Ênfase em reestruturação de Empresas, como requisito à obtenção do título de Especialista, Maringá, 2010.

\author{
1. Professor Doutor Pesquisador do Programa de Pós-Graduação em \\ Gestão e Desenvolvimento Regional da Universidade de Taubaté. \\ Email: jorgeknupp@gmail.com \\ 2. Mestre em Gestão e Desenvolvimento Regional pela Universidade de \\ Taubaté.email: oderlei.santos@gmail.com. \\ 3. Professora Doutora Pesquisadora do Programa de Pós-Graduação em \\ Administração da Pontifícia Universidade Católica de São Paulo. \\ Email: admneusa@pucsp.br
}

